\title{
Bildungsexpansion durch Systemdifferenzierung - am Beispiel der Sekundarstufe II in den 1960er- und 1970er- Jahren
}

\section{Lucien Criblez}

Bildungsexpansion wird im vorliegenden Beitrag nicht in ihren quantitativen (mehr Schülerinnen und Schüler, mehr Schulen, mehr Lehrpersonen, längere Bildungslaufbahnen, höhere Bildungsbeteiligung), sondern in ihren institutionellen Ausprägungen analysiert. Im Zentrum des Interesses steht die Systemdifferenzierung der Sekundarstufe II in den 1960er-und 70er-Jahren. Einerseits werden die Hintergründe dieser Differenzierung ausgeleuchtet, andererseits der Differenzierungsprozess sowohl für die Gymnasien wie auch für die Berufsschulen rekonstruiert. Abschliessend wird gezeigt, dass es sich beim Phänomen Expansion durch Systemdifferenzierung um ein generelles Wachstumsphänomen im Bildungssystem handelt, das nicht nur für die 1960er-und 70er-Jahre und nicht nur für die Sekundarstufe II Gültigkeit hat.

Die Bildungsexpansion der 1960er- und 70er-Jahre wird in der Schweiz ${ }^{1}$ im Allgemeinen mit folgenden Entwicklungen konnotiert: mit dem quantitativen Wachstum der Schülerjahrgänge aufgrund demographischer Entwicklungen (mehr Kinder bedeutet: mehr Schülerinnen und Schüler), mit dem längeren Aufenthalt Jugendlicher und junger Erwachsener im Bildungssystem, also mit der durchschnittlichen Verlängerung von Bildungsbiographien, sowie mit der zunehmenden Öffnung der höheren Bildung für breitere Bevölkerungskreise (allgemein unter dem Schlagwort «Chancengleichheit» diskutiert), die eng mit der Dezentralisierung der Mittelschulen verbunden war. ${ }^{2}$ Der Fokus des folgenden Beitrages liegt auf einer weiteren Entwicklung, die bislang weniger Beachtung fand, die aber wesentlich dafür verantwortlich ist, dass die Expansion über die 1960er- und 70er-Jahre hinaus ihre Folgen hatte, auch nachdem die Schülerjahrgänge wieder geschwunden waren und die Erwartungen, die mit den Postulaten nach "Chancengleichheit» verbunden waren, zumindest was die schichtspezifische Chancengerechtigkeit anbelangt, enttäuscht worden waren. Während die Bildungsexpansion auf der Sekundarstufe I zu einer breiten und bis heute nicht abgeschlossenen ${ }^{3}$ Diskussion über die Struktur dieser Schulstufe führte (verkürzt als "Gesamtschuldiskussion» in Erinnerung) und die Reformabsichten für diese Schulstufe auf Integration, also auf die Aufhebung der Schulty- 
pen oder zumindest die Relativierung der Typendifferenzierung zielten, leitete die Bildungsexpansion auf der Sekundarstufe II in der Schweiz einen Prozess der Systemdifferenzierung ein: Der Maturitätstypus C (mathematisch-naturwissenschaftliche Richtung) wurde den Matutitätstypen A und B (altsprachliches bzw. Latein-Gymnasium) gleichgestellt ${ }^{4}$, zwei neue Maturitätstypen (wirtschaftlicher und neusprachlicher Richtung) wurden eingeführt. Die Diplommittelschule wurde ebenso etabliert wie in einigen Kantonen die sog. "Lehramtsmatur». Zudem konnten der "gebrochene» Bildungsweg stark erweitert und öffentliche Angebote zur Verfügung gestellt werden, die Matur im Erwachsenenalter nachzuholen (sog. Erwachsenenmaturität oder Zweiter Bildungsweg genannt). In den Berufslehren wurden neue Lehrberufe geschaffen und die Berufslehre differenziert: Für begabte Lehrlinge und Lehrtöchter entstand die Berufsmittelschule, als niederschwelliges Angebot die Anlehre.

Im Folgenden werden zunächst die Hintergründe der Bildungsexpansion der 1960er- und 70er-Jahre auf der Sekundarstufe II dargestellt. Es kann davon ausgegangen werden, dass durch das Wirtschaftswachstum, durch die wirtschaftlichen und gesellschaftlichen Veränderungen und die damit verbundenen Probleme der Rekrutierung von wissenschaftlichem und technischem Nachwuchs in den 1960er-Jahren eine ähnliche Situation entstanden war wie in den 1990erJahren auf dem Hintergrund der wirtschaftlichen Globalisierung: Die «Probleme des wissenschaftlichen und technischen Nachwuchses» (Neue Helvetische Gesellschaft, 1961) schürten Ängste, dass die Schweiz im internationalen Wettbewerb nicht bestehen könne, und führten zu einer grundlegenden Kritik am Schulsystem. Auf diesem Hintergrund fand ein starker Ausbau des Bildungssystems auf der Sekundarstufe II und im tertiären Bildungsbereich ${ }^{5}$ statt. Diese ökonomische Dynamik, gekoppelt mit nationalen Existenzängsten im Kalten Krieg, wurde seit spätestens den ausgehenden 1950er-Jahren ergänzt durch eine gesellschaftspolitische Dynamik: Die erstarkende politische Linke forderte eine bessere Beteiligung aller Sozialschichten an den «Früchten» des Wirtschaftswachstums, insbesondere eine bessere Beteiligung am höheren Bildungswesen. Im zweiten Abschnitt wird die Typendifferenzierung des Gymnasiums dargestellt, die einerseits die Rekrutierung neuer Begabungstypen, andererseits begabter Schülerinnen und Schüler, die bisher wegen «sozialer Schranken» (Hess, Latscha \& Schneider, 1966) das Gymnasium nicht besuchten, ermöglichen sollte. Der dritte Abschnitt beschäftigt sich mit einer Art Folgeproblem des Ausbaus der Gymnasien, dass nämlich die Berufsschulen die leistungsstarken Schülerinnen und Schüler zunehmend an die Gymnasien verloren. Auf dem Hintergrund einer Diskussion um die sog. "mittleren Kader» entbrannte eine Diskussion um die Etablierung der Berufsmittelschule (BMS) bzw. der Diplommittelschule (DMS). Aber nicht nur am ,oberen' Ende wurde die Berufslehre differenziert, sondern auch am unteren (Anlehre). Der letzte Abschnitt fragt auf dem Hintergrund der Ausführungen zur Sekundarstufe II nach den Möglichkeiten einer Generalisierung des Phänomens Bildungsexpansion durch Systemdifferenzierung. 


\section{Die Hintergründe der Bildungsexpansion in den 1960 er- und 70er-Jahren}

Die Hintergründe der Bildungsexpansion sind vielfältig und können an dieser Stelle nicht umfassend rekapituliert werden. ${ }^{6}$ Den wichtigsten Hintergrund bildet wohl das lange und ungebrochene Wirtschaftswachstum, das wider Erwarten bald nach dem Zweiten Weltkrieg einsetzte und bis in die frühen 1970er-Jahre anhielt. Dieses Wachstum war gleichzeitig mit einer wirtschaftlichen Umstrukturierung verbunden, in welcher der erste und zweite Beschäftigungssektor zugunsten des dritten an Bedeutung verloren, und in der die in Bildungssystemen erworbenen Qualifikationen wegen des Wandels in der Produktion an Bedeutung zunahmen. Gesellschaftlich war mit diesem Wirtschaftswachstum die Entwicklung hin zum Massenkonsum und zur Massengesellschaft verbunden.

Als zweiter wichtiger Hintergrund kann der Kalte Krieg gelten. Die Bedeutung des Kalten Krieges für die gesellschaftlichen Veränderungen, insbesondere die Veränderung des Bildungssystems, lässt sich am besten am Beispiel des sog. Sputnik-Schocks verdeutlichen. «To a very real extent, history changed on October 4, 1957, when the former Soviet Union successfully launched Sputnik I» - so leitet Roger D. Launius den kürzlich erschienen Sammelband zum SputnikSchock rückblickend ein (Launius et al., 2000, IX). Auf den Schock folgte in den USA eine massive Kritik am Schulsystem, insbesondere am System der höheren Bildung. «Science and education have now become the main battleground of the Cold War. It is upon education that the fate of our way of life depends» (Bonner, 1958, S. 178). Die Übersetzung von Sputnik von einem wissenschaftlichen in ein politisches Ereignis veränderte die Dynamik der Politik - so John A. Douglas in seinem Beitrag mit dem bezeichnenden Titel: «A Certain Future: Sputnik, American Higher Education, and the Survival of a Nation» (Douglas, 2000, S. 328). Die "higher education» wurde als Werkzeug der nationalen Verteidigung entdeckt, und das Schicksal der Nation in Abhängigkeit von der Leistungsfähigkeit des Bildungssystems gebracht ${ }^{7}$. Die Aufmerksamkeit (auch finanzieller Art) für die "higher education» nahm schlagartig zu und die Schulen, Colleges und Universitäten wurden zunehmend durch Gelder des Zentralstaates unterstützt. Diese Entwicklung blieb nicht auf die USA konzentriert. Mit dem üblichen ,time lag' schwappte die Schulkritik nach Europa über. Georg Picht rief in Deutschland die "Bildungskatastrophe» aus (Picht, 1964) und in der Schweiz betitelte der Publizist Alfred A. Häsler einen Sammelband mit Gesprächen über die Schule mit «Schulnot im Wohlstandsstaat» (Häsler, 1967).

Ein dritter Hintergrund: 1961 publizierte die OECD einen Tagungsbericht "Ability and Educational Opportunity", der sich mit Ungleichheiten im Bildungssystem befasste (in Deutsch: OECD, 1967). Mit dem Wirtschaftswachstum und der Entwicklung hin zur Massengesellschaft zeichnete sich also auch ein neues Nachdenken über gesellschaftliche Gerechtigkeit und die Rolle des Bildungssystems im Hinblick auf gesellschaftliche Chancen ab. Dieses Nach- 
denken wurde durch den zunehmenden Mangel an Nachwuchskräften und die damit verbundene Suche nach neuen Begabungspotentialen stark gefördert. Schon der «Arbeitsausschuss zur Förderung des wissenschaftlichen und technischen Nachwuchses» kam 1959 in seinem Schlussbericht zu folgenden Überlegungen:

Wir können die vielfältigen Aufgaben der Förderung des wissenschaftlichen und technischen Nachwuchses nur dann richtig lösen, wenn wir nicht darauf hinausgehen, ein irgendwie zum voraus fixiertes, bestehendes, Reservoir' an Menschen nocheinmal und nocheinmal nach besonderen wissenschaftlichen und technischen Begabungen auszukämmen. Wir müssen etwas ganz anderes tun: Wir müssen die menschliche Basis verbreitern; wir müssen auf einem umfassenden, durch gründliche Erhebungen festgestellten und durch gute Grundschulung vorbereiteten Fundament von Menschen, die zu irgendwelchen qualifizierten Tätigkeiten geeignet sind, aufbauen und jedes Talent und jede Fähigkeit dort einsetzen, wo sie am besten zur Wirkung kommen (Arbeitsausschuss, 1959, S. 6).

Die Ausschöpfung von Begabungsreserven ${ }^{8}$ wurde zum expliziten Ziel der Bemühungen um die Nachwuchsförderung und die ökonomische Absicht wurde zunehmend durch eine gesellschaftspolitische ergänzt: Die «Ungleichheit der Bildungschancen» (Hess, Latscha \& Schneider, 1966) wurde zum Stein des Anstosses, denn «Bildung ist Bürgerrecht» (Dahrendorf, 1965) und sollte deshalb jedem Bürger und jeder Bürgerin zukommen. «Das Recht auf Bildung ist gewährleistet» lautete denn auch der erste Absatz eines neuen Bildungsartikels in der Bundesverfassung, über den aufgrund einer Volksinitiative der Jugendfraktion der Schweizerischen Bauern-, Bürger- und Gewerbepartei ${ }^{9}$ (heute: Schweizerische Volkspartei) abgestimmt wurde. Zwar scheiterte dieser Bildungsartikel in der Volksabstimmung am 4. März 1973 trotz Volksmehr am Ständemehr, aber allein die Tatsache, dass über die Aufnahme eines Rechtes auf Bildung in die Bundesverfassung überhaupt abgestimmt wurde, zeigt die Aktualität der Forderung (Arnet, 2000, S. 17 ff.). ${ }^{10}$

Auf einen weiteren Hintergrund ist hinzuweisen: Das Wirtschaftswachstum, der wissenschaftliche und technologische Wandel sowie die Entwicklung hin zum Sozial- und Wohlfahrtsstaat beförderten eine Grundstimmung der Machbarkeit und der Planbarkeit. Im Bildungsbereich wurden, insbesondere um die Expansion überhaupt bewältigen zu können, Instrumente der Bildungsplanung eingeführt (vgl. den Beitrag von Jürgen Kussau und Lutz Oertel in diesem Heft). Die Bildungsplanung sollte zu einer «rationalen Bildungspolitik» (Widmaier, 1966) führen, Bildungforschung und Bildungplanung wurden als unabdingbare Grundlagen der Bildungspolitik und damit der Reform interpretiert (Widmer, 1976). Auf dem Hintergrund dieser Planungeuphorie - «die Zukunft ist errechenbar» (Stettler, 1994) - wurden zwischen 1959 und 1972 fünf Planungsstudien zum Bedarf von Akademikern und technischem Nachwuchs vorgelegt ${ }^{11}$. Nach dem ersten Bericht des erwähnten Arbeitsausschusses, nach deren Präsident «Bericht Hummler» genannt (Arbeitsausschuss, 1959), erschien 1963 der 
«Bericht der Eidgenössischen Kommission für Nachwuchsfragen auf dem Gebiete der Geisteswissenschaften und der medizinischen Berufe sowie des Lehrerberufes auf der Mittelschulstufe» (Eidg. Kommission, 1963; sog. "Bericht Schultz»), dicht gefolgt vom Bericht der Eidgenössischen Expertenkommission für Fragen der Hochschulförderung (Eidg. Expertenkommission, 1964; sog. "Bericht Labhardt»). Während die ersten beiden Berichte Angebot, Nachfrage und Bedarf an Akademikern und technischem Nachwuchs untersuchten und einen starken Mehrbedarf prognostizierten, konzentrierte sich der «Bericht Labhardt» auf eine Situationsanalyse der Hochschulen. Gefordert wurde hier ein starker Ausbau des schweizerischen Hochschulwesens, denn auch diese Kommission prognostizierte Wachsum, nämlich eine Verdoppelung der Anzahl Studierender bis 1975. Diese Planungsgrundlagen wurden vom 1965 eingesetzten Schweizerischen Wissenschaftsrat mit drei Berichten zum Ausbau der Hochschulen in der Schweiz ergänzt (Schweiz. Wissenschaftsrat, 1967; 1972; 1978). 1971 legte die Arbeitsgruppe Perspektivstudien unter der Leitung von Francesco Kneschaurek im Rahmen der Erarbeitung von Entwicklungsperspektiven für die schweizerische Volkswirtschaft bis zum Jahre 2000 einen Bericht zu den «Perspektiven des schweiz. Bildungswesens» vor (Arbeitsgruppe Perspektivstudien, 1971). «Der Bedarf an Ausgebildeten ist eindeutig grösser als das Angebot auf allen Ausbildungsebenen» (Arbeitsgruppe Perspektivstudien, 1971, S. 128). Der Bericht machte auf diesem Hintergrund auch auf die Dynamik aufmerksam, die zwischen einzelnen Teilen des Bildungssystems, insbesondere in der Berufslehre, in einer Situation der Konkurrenz um den Nachwuchs entstehen könnte (vgl. unten).

Die auf dem Hintergrund der Planungseuphorie entstandene Bildungsökonomie produzierte jedoch nicht immer übereinstimmende Prognosen, wie man dies anhand der erwähnten Berichte annehmen könnte. Die 1972 von einer Gruppe um Matthias Jermann publizierte Planungsstudie kam zu einem anderen Schluss: «Die aus dem Bildungsmodell berechneten Abgänge von Techniken und Hochschulen werden reichlich genügen, um den aus der Strukturprognose abgeleiteten Bedarf zu decken» (Jermann et al., 1972, S. 249). Aber nicht so sehr die widersprüchlichen Prognosen, sondern vor allem das Ende der langen wirtschaftlichen Prosperität verwandelten die Planungseuphorie in der Mitte der 1970er-Jahre zunehmend in Planungskritik.

Als Ausgangspunkt der Bildungsexpansion in der Schweiz kann also u.a. die Sorge um den technischen und wissenschaftlichen Nachwuchs gelten. Immer wieder wurde vergleichend argumentiert, dass die Schweiz im Bereich der höheren Bildung gegenüber andern Ländern in einen Rückstand geraten sei. Der Sputnikschock kristallisierte diese Sorgen - nicht nur in den USA, sondern auch in Deutschland und der Schweiz - zum «political symbol and tool» (Van Dyke, 2000), und schuf einen optimalen Legitimationshintergrund für die Bildungsexpansion, die auf dem Hintergrund einer technologischen Planungseuphorie als plan- und steuerbar galt. Obwohl die Expansion zunächst auch eine Folge grös- 
serer Schülerjahrgänge war, womit ein Wachstum des gesamten Bildungssystems verbunden war, und obwohl vom Kindergarten über die Stipendienpolitik bis hin zur sog. "éducation permanente» alle Bildungsbereiche von Reformen betroffen waren, galt die höhere Bildung, insbesondere die universitäre Bildung und deren Basis, die Gymnasien, als Kernzone der Expansion. Durch die Bildungsexpansion sollte explizit die Basis für den Nachwuchs verbreitert werden. Die wichtigsten Reformen mussten deshalb in den Gymnasien erfolgen.

\section{Typendifferenzierung des Gymnasiums}

Bereits im «Bericht Hummler» (vgl. oben) war gefordert worden: «Der Pflege des naturwissenschaftlichen Unterrichts an den Mittelschulen und der Frage der Gleichbehandlung der Maturitätstypen ist besondere Aufmerksamkeit zu schenken» (Arbeitsausschuss, 1959, S. 50). Damit wurde eine alte Forderung wieder aufgenommen. Seit der Einführung der Maturitätstypen A, B, und C in der eidgenössischen Maturitäts-Anerkennungsverordnung von 1925 war nämlich immer wieder gefordert worden, dass die C-Matur (das mathematisch-naturwissenschaftliche Gymnasium) den beiden andern Maturitätstypen gleichzustellen sei, konkreter: ohne zusätzliche Lateinauflagen zum Zugang zu den eidgenössischen Medizinalprüfungen führen solle.

Von Seiten der Gymnasien war dies eine alte Forderung ${ }^{12}$, hatte doch zum Beispiel der Rektor der Basler Töchterschule, Albert Barth, bereits 1919 ein Gutachten vorgelegt, in dem er ein altsprachliches, ein neusprachliches und ein mathematisch-naturwissenschaftliches Gymnasium und die Gleichbehandlung aller drei Typen gefordert hatte (Barth, 1919). In einer Analyse der Situation des Gymnasiums («Gegenwartsfragen des Gymnasiums») kam die Konferenz der schweizerischen Gymnasialrektoren (KSGR) kurz nach dem Zweiten Weltkrieg ebenfalls zum Schluss, dass der Maturitätstyp C gleichberechtigt zum allgemeinen Universitätszugang führen sollte (KSGR, 1948). Da aber nicht mehr allein die Frage nach den bisherigen Typen und deren Berechtigung zur Diskussion stand, sondern die Basis für die Nachwuchsrekrutierung in die höhere Bildung verbreitert werden sollte, setzte die KSGR 1958 eine Studienkommission für Typenfragen ein, deren Bericht wiederum die Gleichstellung des Maturitätstypus C forderte (Studienkommission für Typenfragen, 1961). Trotz heftigster Widerstände der Mediziner, welche die Zugangsbeschränkung zum Medizinstudium durch die Lateinauflage immer als wichtigen Teil ihrer Standespolitik interpretiert hatten, und der eidgenössischen Maturitätskommission konnte die Gleichberechtigung des Maturtypus $\mathrm{C}$ hinsichtlich der Zulassung zu den eidgenössischen Medizinalprüfungen in der Maturitäts-Anerkennungsverordnung vom 22. Mai 1968 endlich realisiert werden. Gleichzeitig fand auch der zweite Bildungsweg formal erstmals seine Anerkennung, und die Vorschriften über die Anerkennung des sog. gebrochenen Bildungsweges (Eintritt ins Gymnasium 
nach der Sekundar- bzw. der Bezirksschule) wurden gelockert. Mit der letzteren Veränderung wurden die Möglichkeiten erweitert, den auf die Maturität vorbereitenden Unterricht während der obligatorischen Schulzeit, vor allem im 7. und 8. Schuljahr, vermehrt dezentral an Sekundar- bzw. Bezirksschulen anzubieten. Damit sollte insbesondere Jugendlichen ausserhalb der städtischen Agglomerationen der Zugang zur höheren Bildung erleichtert werden.

Schon während der Diskussion um die Reform der Maturitäts-Anerkennungsverordnung (MAV) 1968 war über die Einführung weiterer Maturitätstypen diskutiert worden. Die KSGR hatte bereits in ihrem Bericht 1961 auf weitere Typen hingewiesen, die eine Anerkennung anstrebten. Der Zeitpunkt war aber als zu früh erachtet worden, und die Reform schien mit der Öffnung des lateinlosen Zugangs zu den Medizinalprüfungen politisch hinreichend belastet. Nachdem die Durchsetzung dieser Reform über 40 Jahre gedauert hatte, er-staunt es, dass die nächste Revision der MAV bereits Ende 1972 vollzogen wurde.

Der im ersten Abschnitt dargestellte gesellschaftspolitische Hintergrund der Bildungsexpansion hatte bereits die Durchsetzung des Postulates nach Gleichstellung des Maturitätstypus $\mathrm{C}$ erleichtert. Die Forderung nach der Rekrutierung neuer Begabungspotentiale ermöglichte nun auch die Einführung der Maturitätstypen D und E. Ausgelöst wurde die Reform durch offiziell eingereichte Gesuche für die Anerkennung von neusprachlichen Gymnasien und Wirtschaftsgymnasien (Egger, 1978, S. 120 ff.). Obwohl bildungspolitisch weitgehende Einigkeit darüber bestand, dass eine allzu starke Differenzierung der Maturitätsschulen nicht sinnvoll sei, da das Maturitätszeugnis weiterhin als allgemeines Hochschulzugangszeugnis gelten und nicht irgendeine ,Fakultätsreife' bezeugen sollte, konnte die Reform schnell realisiert werden. Dies hat unterschiedliche und komplexe Gründe, vier der wichtigsten sollen erwähnt werden: Erstens war der Druck, die gymnasiale Basis für den wissenschaftlichen und technischen Nachwuchs im beschriebenen Sinne zu erweitern, Ende der 1960erund anfangs der 1970er Jahre sehr gross geworden. Die beiden neuen Maturitätstypen sollten die Rekrutierung neuer Begabungstypen und von Jugendlichen aus bildungsferneren Bevölkerungsschichten ermöglichen. Zweitens stand mit der Revision des Bildungsartikels in der Bundesverfassung (vgl. oben) in Aussicht, dass die enge verfassungsmässige Bindung der Maturitäts-Anerkennungsverordnung an die Zulassung zu den Medizinalprüfungen erweitert würde. Der Widerstand der Mediziner wurde dadurch stark relativiert. Drittens wurde parallel zur MAV-Revision der Bericht der Expertenkommission «Mittelschule von morgen» erarbeitet und 1972 vorgelegt (Expertenkommission, 1972), dessen Vorschläge für eine Reform der Mittelschulen wesentlich weiter gingen als diejenigen zur Revision der Maturitäts-Anerkennungsverordnung. Als weiterer Grund für die schnelle Einführung der beiden neuen Maturitätstypen muss beachtet werden, dass sowohl für das neusprachliche Gymnasium wie für das Wirtschaftsgymnasium bereits mehrere kantonale Vorbilder existierten ( 4 bzw. 16 Schulen) und dass für beide auch elaborierte Begründungen bzw. Kon- 
zepte vorlagen. Für das Wirtschaftsgymnasium hatte zum Beispiel Rolf Dubs auf der Grundlage des sog. «Käfer-Plans» ${ }^{13} 1968$ ein ausführliches Konzept vorgelegt (Dubs, 1968).

Die Reformen verpassten ihre Wirkung in quantitativer Hinsicht nicht. Die folgende Tabelle gibt einen Überblick über die Veränderung der Anzahl angebotener Ausbildungsgänge (= Summe der pro Standort angebotenen Maturitätstypen) und der Anzahl Maturitätsschulen zwischen 1968 und 1983. Insgesamt hat sich die Anzahl der angebotenen Ausbildungsgänge mehr als verdreifacht, die Anzahl anerkannter Maturitätsschulen mehr als verdoppelt. Die Anzahl der Schülerinnen und Schüler im 10.-13. Schuljahr der schweizerischen Gymnasien verdoppelte sich zwischen 1970 und 1982 (Meylan \& Ritter, 1985, S. 37). Quantitativ können die Reformen der Maturitäts-Anerkennungsverordnung 1968 und 1972 im Hinblick auf die Öffnung der Gymnasien, auf die Rekrutierung neuer Begabungspotentiale und auf die Förderung des wissenschaftlichen Nachwuchses im Rückblick als Erfolg bezeichnet werden.

Tabelle 1: Ausbau und Typendifferenzierung der Gymnasien in der Schweiz, 1968-1983 (Meylan \& Ritter, 1985, S. 37-39)

\begin{tabular}{|l|c|c|}
\hline & 1968 & 1983 \\
\hline Angebot an Ausbildungsgängen Typ A & 48 & 95 \\
\hline Angebot an Ausbildungsgängen Typ B & 51 & 85 \\
\hline Angebot an Ausbildungsgängen Typ C & 33 & 52 \\
\hline Angebot an Ausbildungsgängen Typ D & - & 48 \\
\hline Angebot an Ausbildungsgängen Typ E & 132 & 391 \\
\hline$\sum$ angebotener Ausbildungsgänge & 57 & 129 \\
\hline Anzahl anerkannter Maturitätsschulen & - & 4 \\
\hline
\end{tabular}

Als weitere Differenzierung des gymnasialen Angebots kann die Ausweitung des sog. «zweiten Bildungsweges» gelten. Am 27. September 1961 begründete Nationalrat Haller ein Postulat, das die Förderung des zweiten Bildungsweges konkreter: die Änderung der Artikel 13 und 14 der Eidgenössischen MaturitätsAnerkennungsverordnung - verlangte, wie folgt:

Zwei Wege zur Abhilfe [des chronischen Mangels an wissenschaftlichen und technischen Mitarbeitern] stehen uns zur Verfügung und könnten bei grosszügigerer Handhabung zu positiven Resultaten führen. Der erste Weg ist der, dass auch aus Kreisen der unteren Erwerbsschichten vermehrt fähigen jungen Leuten der $Z u$ gang zu den akademischen Berufen ermöglicht wird. Der zweite Weg besteht darin, dass auch Leuten, die schon im Berufsleben stehen, also eine gewerbliche oder kaufmännische Lehre absolviert haben, der Zugang zu den Hochschulen offenstehen soll. Diese zweite Art der Möglichkeit zur Vorbereitung auf das Hoch- 
schulstudium kennt man im Ausland unter dem Namen "zweiter Bildungsweg». [...] Der Kanton Basel-Stadt kennt seit 1931 Abendkurse für Erwerbstätige zur Erlangung der Maturität (zitiert nach der Zeitschrift «Nachwuchsförderung» Nr. 1 [1962], 34).

Die Argumente für einen solchen zweiten Bildungsweg zur Förderung des wissenschaftlichen und technischen Nachwuchses sind im Rückblick selbstredend. Tatsächlich wurde der Schaffung vermehrter Möglichkeiten im Sinne des zweiten Bildungsweges (z.B. Erwachsenenmaturität, Abendtechnikum) und insbesondere der staatlichen Unterstützung solcher Bemühungen ein wichtiger Stellenwert im Hinblick auf die Ausschöpfung von Begabungsreserven und die Nachwuchsförderung zugeordnet (Durtschi, 1963; Schaub, 1966). Die MAV vom 22. Mai 1968 schuf denn auch erstmals die Möglichkeit, die Erlangung der Maturität auf dem zweiten Bildungsweg formal anzuerkennen.

Die zweimalige Revision der MAV innerhalb kurzer Zeit hatte aber noch nicht alle Reformabsichten realisiert. Zumindest drei Probleme standen weiterhin zur Lösung an. Zunächst blieb die Frage der Anerkennung weiterer Maturitätstypen, insbesondere diejenige eines musischen Gymnasiums, diejenige eines pädagogischen Gymnasium als ,Unterbau' für eine Lehrerbildung auf Tertiärstufe und diejenige des Sportgymnasiums offen. Zweitens war die Rückkehr zu einer typenlosen Matur mit der Möglichkeit eines obligatorischen Wahlbereichs bereits für die MAV-Revision 1972 in Erwägung gezogen, jedoch nicht realisiert worden. Die Schweizerische Konferenz der kantonalen Erziehungsdirektoren hat diese Idee, wenn auch abgeschwächt, in ihre Empfehlungen zum Bericht «Mittelschule von morgen» aufgenommen, indem sie ihrer Kommission für Mittelschulfragen den Auftrag erteilte abzuklären, «in welchem Sinne und auf welche Weise eine Reduktion bzw. eine Konzentration der Anzahl der Maturitätstypen eingeleitet werden könnte» (EDK, 1976, S. 37). Drittens blieb die Struktur des Gymnasiums und die Funktionsteilung innerhalb der Sekundarstufe II ungeklärt. Zwar war mit den Reformen und der Öffnung des Gymnasiums eine Entwicklung vom Elitegymnasium zum Massengymnasium eingeleitet worden (Criblez, 2000b), aber zu einer vierjährigen Maturitätsstufe mit vorgängiger Beobachtungs- und Orientierungsstufe unter Einführung einer parallelen dreijährigen Diplomstufe, wie dies von der Expertenkommission «Mittelschule von morgen» vorgeschlagen worden war (Expertenkommission, 1972), konnte sich die EDK in den erwähnten Empfehlungen nicht durchringen. Erst die Revision der Maturitäts-Anerkennungsverordnung von 1995 sollte zumindest die beiden ersten Postulate einlösen (Meylan, 1996).

«Volksschule oder Selektionsschule?» - so beschrieb der Zürcher Erziehungsrat 1964 die alternativen Entwicklungsperspektiven für das schweizerische Gymnasium. Er kam zum Schluss, dass der Nachholbedarf an wissenschaftlichem und technischem Nachwuchs nur aufzuholen sei, wenn sich «unsere Mittel- und Hochschulen in den Dienst der grossen Zahl stellen und neue Wege in der Schulpolitik» beschreiten (Erziehungsrat, 1964, S. 99). Diskutiert wurden auf 
diesem Hintergrund insbesondere die aus damaliger Sicht nicht mehr zeitgemässen Selektionspraktiken, die Abschaffung der Mittelschultypen zugunsten einer Einheitsmatur und der Umbau des Gymnasiums zu einer Volksschule. Der Prozess hin zur "grossen Zahl» ist insbesondere durch die Bildungsexpansion gefördert worden. Aber man war sich damals auch mehrheitlich einig, dass das amerikanische High School-Modell den schweizerischen Verhältnissen nicht angepasst sei. Eine Studiengruppe der Konferenz Schweizerischer Gymnasialrektoren hatte zwar die amerikanischen High Schools studiert (Studienkommission, 1965), aber die Frage, die Ludwig Räber im Anschluss stellte: «Ist unser traditionelles Gymnasium abbruchreif, und soll an seiner Stelle eine ,High School' schweizerischer Prägung entstehen?» (Räber, 1969, S. 103), war durchaus rhetorisch gemeint. Denn - so Rolf Deppeler - die High School sei nicht besser als unsere Gymnasien, sie sei anders (Deppeler, 1967, S. 191).

\section{Eine Schule für mittlere Kader: Berufsmittel- schule oder Diplommittelschule?}

Bereits während der Mittelschul-Expansionsphase der 1960er-Jahre wurde ein Diskurs über die möglichen institutionellen und qualitativen Folgen der Expansion geführt. So legte zum Beispiel der ETH-Professor Otto Zweifel 1967 im "gymnasium helveticum» Überlegungen zu "Gefahren der Qualitätseinbusse bei ansteigender Maturandenquote» vor (Zweifel, 1967). An anderer Stelle, in der «Schweizerischen Zeitschrift für Nachwuchs und Ausbildung», wurde über die «Bildungsinflation am Gymnasium» (Faerber, 1966), über die Frage, ob die Expansion das Bildungsniveau sinken lasse (Panchaud, 1966) oder allgemein über die Frage, ob die höhere Ausbildung überschätzt werde (Hess, 1966), sinniert. Alle Bedenken wurden mit guten Gründen verworfen. Aber über eine Folge der Expansion war lange zu wenig nachgedacht worden: Dass die Expansion nämlich Folgen für die anderen Ausbildungsbereiche der Sekundarstufe II, insbesondere für die Berufsbildung haben könnte.

Im September 1967 publizierte Lajos Nyikos, Rektor des Gymnasiums am Kohlenberg in Basel und Präsident einer vom Vorstand des Vereins Schweizerischer Gymnasiallehrer (VSG) eingesetzten Arbeitsgruppe, im Namen dieser Arbeitsgruppe im "gymnasium helveticum» einen Beitrag mit dem Titel «Eine Schule für mittlere Kader?» (Nyikos, 1967) ${ }^{14}$. In sechs Thesen stellte er die Grundlinien eines neuen Schultyps auf der Sekundarstufe II dar, der zwischen Gymnasium und Berufsbildung angesiedelt sein sollte. Das Bedürfnis der Wirtschaft für besser ausgebildete Kader sei ausgewiesen, und die traditionelle Berufsbildung genüge den Anforderungen einer «wachsenden Intellektualisierung der Berufe» nicht mehr (Nyikos, 1967, S. 33). Die forcierte Förderung des akademischen Nachwuchses durch die Gymnasien erschwere zunehmend die Rekrutierung des qualifizierten Technikernachwuchses. Es sei deshalb höchste Zeit, 
die Bemühungen um höhere Bildung nicht ausschliesslich auf die Gymnasien und die zukünftigen Akademiker zu konzentrieren. Aber gleichzeitig genüge der Weg über die Volksschule und die traditionelle Berufslehre vielen Berufen heute nicht mehr. Eine Matur sei aber für diese Berufe nicht unbedingt notwendig oder sogar nicht erwünscht. "Wir postulieren nicht das Gymnasium für alle» (Nyikos, 1967, S. 36).

Als Vorbild dienten Nyikos das Unterseminar und die Diplomhandelsschule. Die neue Schule sollte an die obligatorische Volksschule anschliessen, zwei bis vier Jahre dauern und sowohl eine allgemeine wie auch eine vorberufliche Ausbildung bieten. Neben obligatorischen Kernfächern sollte die Ausbildung obligatorische Wahlfächer, insbesondere berufstypische Fächer und Praktika, sowie eine Reihe von Freifächern umfassen. Das verliehene Diplom sollte zum Übertritt in eine weiterführende Berufsausbildung oder direkt ins Berufsleben berechtigen.

Im Folgenden präzisierte und modifizierte Nyjkos diese Idee (Nyikos, 1968a; 1968 b) - so änderte er zum Beispiel den Namen in «Berufsmittelschule» ab -, die Grundidee einer solchen neuen Schule auf der Sekundarstufe II war aber lanciert und entwickelte eine interessante Dynamik zwischen unterschiedlichen Akteuren der Sekundarstufe II.

Nur einige Monate nach der Veröffentlichung des ersten Beitrages von Nyikos waren die Vorschläge für eine «Schule für mittlere Kader» bzw. für die Berufsmittelschule Hauptthema an der Delegiertenversammlung des Schweizerischen Verbandes für Gewerbeunterricht am 10. Februar 1968 in Zürich. Die Delegierten «sahen in diesem Projekt eine schwere Bedrohung der Lehre und einen Übergriff der Gymnasiallehrer auf das Hoheitsgebiet der Berufsbildung» (Sommerhalder, 1989, S. 55). Was war geschehen? Die Gewerbelehrerschaft war von der Idee des neuen Schultyps in mehrfacher Hinsicht aufgeschreckt worden. Zunächst war die Idee von den Gymnasialrektoren und vom Verein Schweizerischer Gymnasiallehrer entwickelt worden, also von der «Konkurrenz» (die bislang keine Konkurrenz war, weil man sich gegenseitig kaum wahrnahm). Zweitens vermutete man, dass das Gymnasium, das trotz Nachwuchsmangels hohe Selektionsraten beibehalten hatte, sich seines schlechten Gewissens gegenüber den hinausselektionierten Schülerinnen und Schülern entledigen wollte, indem für diese Gruppierung ein neuer Schultyp geschaffen werden sollte. Die Schülerinnen und Schüler, die aus Sicht des Gymnasiums nicht ins Gymnasium gehörten, sollten mit einem attraktiven Angebot vom Gymnasium ferngehalten werden. Nyikos bemängelte nämlich, dass es im «heutigen Schulsystem nur eine Art von ,Durchlässigkeit'» gäbe, "die wirklich funktioniert: den Sturz ,von oben nach unten'» (Nyikos, 1968a, S. 101).

Weiter störte die Gewerbelehrerschaft, dass Nyikos als Vertreter der Gymnasien die Berufslehre kritisierte: Die geringe schulische Weiterqualifikation durch die Berufslehre genüge den Anforderungen vieler Berufe, insbesondere eben dem mittleren Kader, nicht mehr. Das Bundesgesetz über die Berufsbildung ${ }^{15}$ war 
zwar zu Beginn der 1960er Jahre einer Revision unterzogen worden, wesentliche Probleme konnten dabei jedoch nicht gelöst werden und die Berufsbildung schien weiter reformbedürftig (vgl. etwa Chresta, 1970; Dellsperger, 1968; Sommerhalder, 1970). So beklagte die Gewerbelehrerschaft selber die mangelnden Laufbahnperspektiven für die Lehrabgänger (die Ausbauphase der höheren Fachschulen, insbesondere im technischen und administrativen Bereich stand erst bevor) sowie die fehlende Differenzierung der Berufslehre. "Das Eintopfgericht muss einem differenzierten Angebot weichen!» (Sommerhalder, 1989, S. 39). Die Berufslehre hatte zudem - ähnlich wie die Gymnasien - seit den 1950er-Jahren einen starken Ausbau erfahren. Im Rahmen der wirtschaftlichen Prosperität waren immer mehr und immer spezifischere Lehrberufe geschaffen worden, um Jugendliche für die Berufslehre gewinnen zu können. Die Ausbildung auf der Sekundarstufe II wurde immer mehr zum normalen Bildungsweg aller Jugendlicher. Die Anzahl der Lehrlingsausbildungsplätze verdoppelte sich zwischen 1950 und 1970 (Feierabend, 1975, S. 25). Dies bedeutete, dass immer mehr Jugendliche, die früher von ihren intellektuellen Leistungsfähigkeiten her nie eine Berufslehre absolviert hatten, in die Berufslehren drangen. Dies hatte u.a. zur Folge, dass die Durchfallquoten bei den Lehrabschlussprüfungen gestiegen waren (Sommerhalder, 1968, S. 226 ff.). Gleichzeitig sorgte der Sog in die Gymnasien dafür, dass den Gewerbeschulen die fähigsten Jugendlichen entzogen wurden. Die sog. ,Auspowerung' der Berufsbildung durch Abwanderung ins Gymnasium war eine der grössten Sorgen der Gewerbelehrerschaft - und offensichtlich hatte auch die Gymnasiallehrerschaft dieses Problem erkannt.

Ein Teil der Gewerbelehrerschaft hatte schon verschiedentlich Reformen vorgeschlagen, war damit bislang jedoch nicht durchgedrungen. Erst gegen Ende der 1960er Jahre wurden die Probleme immer virulenter und die Stimmen, die weitergehende Reformen verlangten, immer lauter. Der Vorstoss aus Kreisen der Gymnasien für eine «Schule für mittlere Kader» wirkte deshalb wie ein Katalysator. Zwar widersetzte sich die Gewerbelehrerschaft an ihrer Delegiertenversammlung den Ideen der Gymnasiallehrerschaft vehement, aber nicht, weil die Kritik an der Berufsbildung als völlig unhaltbar erschien, und auch nicht, weil die Grundidee völlig aus der Luft gegriffen schien. Ein ,Minigymnasium' wollte man aber nicht, und die mittleren Kader sollten auch in Zukunft nicht im praxisfernen Schulraum, sondern im dualen System der Berufsbildung ausgebildet werden. "Das mittlere Kader steht mit der Arbeiter- und Angestelltenschaft meist in unmittelbarem Kontakt, und da sind fundierte Kenntnisse über die menschliche und berufliche Situation der Untergebenen vonnöten, wenn die Führung erfolgreich sein will» (Grossenbacher, 1968, S. 105). Eine neue Schule, die sich zum Ziele setze, das mittlere Kader auf Kosten einer gründlichen Berufsausbildung heranzubilden, wurde abgelehnt, auch wenn die Idee, jungen Leuten eine bessere Allgemeinbildung zu vermitteln, grundsätzlich akzeptiert war. «Man war gemeinsam empört, dass die ohnehin privilegierten Gymnasiallehrer, deren Schulen im ganzen Lande in einer einmalig grosszügigen Ausbauphase standen, 
sich einen weiteren Schulzweig angliedern wollten, und dies auf Kosten der benachteiligten Gewerbeschule» (Sommerhalder, 1989, S. 62). Die Berufsmittelschule, so der allgemeine Tenor bei der Gewerbelehrerschaft, sollte an der Gewerbeschule angesiedelt werden.

Die Gewerbelehrerschaft übernahm nun die Initiative: Im Frühjahr 1968 setzten der Schweizerische Verband für Gewerbeunterricht (SVGU) und die Schweizerische Direktorenkonferenz gewerblicher Berufs- und Fachschulen (SDK) eine Kommission ein, welche sich der anstehenden Probleme annehmen sollte. Parallel dazu beauftragten auch die politischen Instanzen, das eidgenössische Volkswirtschaftsdepartement, eine Expertenkommission (sog. Kommission Grübel), die mit der Erarbeitung von Vorschlägen für die Verbesserung der Berufslehre beauftragt wurde. Dieses Mandat führte letztlich zum Bundesgesetz über die Berufsbildung vom 3. Dezember 1978 (Feierabend, 1975, S. 31 ff.).

Während der politische Prozess der Gesetzesrevision längere Zeit dauerte, kam die Studienkommission von SVGU und SDK sehr rasch zu ersten Ergebnissen: Sie verlangte, dass die Berufslehre in eine Anlehre bzw. Kurzlehre, in eine Normallehre, deren Unterricht jedoch erweitert werden sollte, und in eine Berufsmittelschule zu differenzieren sei (Sommerhalder, 1968; Studienkommission, 1968). Institutionell überstürzten sich die Entwicklungen dann ${ }^{16}$, wenn man das übliche Reformtempo in der schweizerischen Bildungspolitik als Vergleichsmassstab nimmt. Im Herbst 1968 eröffnete die Gewerbeschule Aarau versuchsweise die erste gewerbliche Berufsmittelschule (BMS) der Schweiz, im Frühjahr 1969 folgte Bern, wo seit 1972 auch gestalterische Klassen geführt wurden. Im Herbst 1970 eröffnete die Gewerbeschule der Stadt Zürich ihre BMS, im Frühjahr 1972 folgte eine BMS in Muttenz (Basel-Landschaft) und 1972 eröffnete die Handelsschule des Kaufmännischen Vereins Zürich die erste BMS im kaufmännischen Bereich.

Mit der «Wegleitung für die Errichtung und die Organisation von Berufsmittelschulen und deren Subventionierung durch den Bund» vom 26. Juni 1970 definierte das Bundesamt für Industrie, Gewerbe und Arbeit (BIGA) die Berufsmittelschule als Teil der im Berufsbildungsgesetz vorgesehenen freiwilligen Kurse und legte ihr Ziel fest: «Die Berufsmittelschule vermittelt geeigneten Lehrlingen und Lehrtöchtern aller Berufe als Ergänzung zum Pflichtunterricht an der Berufsschule eine breitere theoretische Schulung zur Vertiefung der beruflichen Ausbildung und zur Erweiterung der allgemeinen Bildung» (Wegleitung 1970, Abs. 1). Die Wegleitung hatte provisorischen Charakter; sie sollte ermöglichen, erste Erfahrungen zu sammeln. Diese Erfahrungen waren durchaus positiv $^{17}$, kommt doch die Botschaft des Bundesrates zum Bundesgesetz über die Berufsbildung zum Schluss: «Die seinerzeit etwas improvisiert eingeführte Berufsmittelschule hat sich bewährt und ist für nicht wenige Lehrlinge eine gute Alternative zur Mittelschule» (Botschaft Berufsbildungsgesetz, 1977, S. 710). So wurde die Berufsmittelschule innerhalb kurzer Zeit eingeführt und später mit dem neuen Berufsbildungsgesetz institutionalisiert. Ähnlich erging es dem An- 
liegen der Anlehre. Auch sie wurde mit dem neuen Berufsbildungsgesetz definitiv durch Bundesgesetzgebung geregelt. Die Bildungsexpansion betraf so auf der Sekundarstufe II sowohl die Mittelschulen als auch die Berufsschulen. Im Bereich der Berufsmittelschule kann sogar davon ausgegangen werden, dass deren Einführung eine erste Folge der Expansion der Gymnasien war.

Hatte sich damit die Gewerbelehrerschaft gegenüber der Gymnasiallehrerschaft durchgesetzt? In gewisser Hinsicht ja - die BMS wurde an den Berufsschulen realisiert. Daneben aber entstand - vor allem auf Berufe im pädagogischen, sozialen und Gesundheitsbereich ausgerichtet - auch eine neue Mittelschule auf schulischer Basis, die durchaus als Realisierung der Ideen der Gymnasiallehrkräfte und der Gymnasialrektoren gelten kann: die Diplommittelschule (DMS).

Nachdem der Verein Schweizerischer Gymnasiallehrer die Vorschläge für «eine neue Schule für mittlere Kader» bereits an seiner Generalversammlung in Genf im Oktober 1967 unterstützt hatte, und nachdem die «Expertenkommission zum Studium der Mittelschule von morgen» 1972 in ihrem Bericht empfohlen hatte, neben der Maturitätsstufe eine Diplomstufe einzuführen (Expertenkommission, 1972), mussten die konzeptionellen Arbeiten für die DMS trotz Konkurrenz durch die Berufsmittelschule weiter vorangetrieben werden. Der Vorstand der EDK setzte im April 1975 eine «Studienkommission Diplommittelschulen» ein. Diese legte zwei Jahre später einen Bericht vor, in dem sie nicht nur die erwarteten Grundlagen für die DMS zur Verfügung stellte, sondern auch darauf hinwies, dass in der Schweiz bereits 30 Diplommittelschulen existierten. Insbesondere die früheren allgemein bildenden Fortbildungsschulen für Mädchen waren zu Diplommittelschulen weiterentwickelt worden (Studienkommission Diplommittelschulen, 1977, S. 83-85). Die EDK reduzierte die von der Studienkomission vorgeschlagenen sechs Modelle anschliessend auf zwei, eine Ad hoc-Arbeitsgruppe erarbeitete aber 1981 ein neues Modell mit zwei Varianten, einer zwei- und einer dreijährigen DMS, die auf drei Berufsfelder ausgerichtet sein sollten, auf paramedizinische, sozial-erzieherische und adminstrative Berufe (Ad hoc-Arbeitsgruppe, 1983, S. $31 \mathrm{ff.}$.).

Der Konflikt zwischen Gymnasiallehrkräften und der Gewerbelehrerschaft um die «Schule für mittlere Kader» war also nicht zugunsten der einen Seite entschieden worden, sondern führte sowohl zur Einführung der Berufsmittelschule wie auch zur Etablierung der Diplommittelschule.

\section{Bildungsexpansion durch Systemdifferenzierung - einige Schlussfolgerungen}

Die Systemdifferenzierung ist neben dem quantitativen Ausbau (grössere Schülerjahrgänge, mehr Lehrerkräfte, mehr Schulen), längeren Bildungsbiographien bzw. längerem Aufenthalt im Bildungssystem und höherer Bildungsbeteiligung 
eine der grundlegenden Erscheinungen der Bildungsexpansion. Sie ist wesentlich dafür verantwortlich, dass die Expansion auch über die Phase der grossen Schülerjahrgänge hinaus anhielt. Im Hinblick auf die Expansion des gesamten Bildungssystems und unabhängig von der Bildungsexpansionsphase der 1960erund 70er Jahre stellen sich zwei Generalisierungsfragen, die über das hier behandelte Beispiel der Sekundarstufe II hinausweisen: Entspricht der am Beispiel der Sekundarstufe II gezeigte Mechanismus von Bildungsexpansion durch Systemdifferenzierung einem generellen Entwicklungsmuster im Bildungssystem? Wenn dies zutrifft, müsste er sich auch für andere Bildungsstufen nachweisen lassen. Und wie verhält sich der Prozess der Systemdifferenzierung zum gegenläufigen Prozess, zur Integration bzw. zur Aufhebung von Schultypen, der zum Beispiel für die Sekundarstufe I während der Bildungsexpansionsphase im Rahmen der Gesamtschuldiskussion eingeleitet wurde?

Im Folgenden soll zunächst gezeigt werden, dass sich der Prozess der Bildungsexpansion durch Systemdifferenzierung tatsächlich als generelles Wachstumsphänomen für das schweizerische Bildungssystem beschreiben lässt. Drei Beispiele mögen dies dokumentieren:

Nach 1830 wurden als weiterführende Schulen die Sekundar- oder Bezirksschulen eingerichtet. Solange die Schülerinnen und Schüler, die keine weiterführenden Schulen besuchten, die Schule nach der Primarschule verliessen, blieb das System von Primar- und Sekundarschulen konsistent. Mit der allmählichen Verlängerung der Unterrichtspflicht auf neun Jahre für alle Schülerinnen und Schüler entstand aber neben den Sekundar- bzw. Bezirksschulen eine Primaroberstufe. In einzelnen Kantonen wurde dieses zweigliedrige System im Verlaufe des 20. Jahrhunderts sogar zu einem dreigliedrigen differenziert (z.B. in den Kantonen Zürich, Aargau und Solothurn). Diese Differenzierung war in den 1950er-Jahren weitgehend abgeschlossen. An diese Differenzierungsphase der Sekundarstufe I schliesst sich seit den 1960er-Jahren eine Phase der Integration an: Mit der Gesamtschuldebatte bzw. der Diskussion über integrierte und kooperative Schulmodelle wurden die Schultypen auf der Sekundarstufe I im Verlaufe der letzten 30 Jahre immer mehr relativiert (Jenzer, 1998, S. 74 ff.).

Die Bildungsexpansion hatte nicht nur einen Ausbau der höheren Bildung im gymnasialen und universitären Bereich gefördert, sondern führte zum Ausbau bzw. zur Gründung von neuen Institutionen im tertiären Bildungsbereich. Die Anzahl Technika wurde stark erweitert, im kaufmännischen Bereich wurden Höhere Wirtschafts- und Verwaltungsschulen aufgebaut und weitere Berufsbereiche folgten mit Höheren Fachschulen (Landwirtschaft, Gestaltung, Sozialpädagogik, Sozialarbeit usw.). Es entstand ein differenziertes System von Höheren Fachschulen, das seit Beginn der 1990er-Jahre in ein Fachhochschulsystem transformiert wird. Dieser Transformationsprozess kann als Integrationsprozess interpretiert werden. Damit ist jedoch gleichzeitig eine Differenzierung des Hochschulsystems in universitäre Hochschulen und Fachhochschulen verbunden. Aber nach dieser Differenzierung zeichnet sich bereits die Integration ab. 
Die neu entstandenen Fachhochschulen werden im Vergleich mit den traditionellen Hochschulen als "gleichwertig, aber andersartig» (Botschaft Fachhochschulgesetz, 1994, S. 16) charakterisiert. Dass die beiden Institutionstypen Universität und Fachhochschule einander angenähert werden sollen, zeigt auch das aktuell zentrale Grundlagendokument der schweizerischen Bildungspolitik, die «Botschaft über die Förderung von Bildung, Forschung und Technologie in den Jahren 2000-2003»: «Der Leitgedanke für die künftige Hochschulpolitik des Bundes ist die Einheit des Tertiärbereiches» (Botschaft Bildung, 1998, S. 19; Hervorhebung im Original).

Letztlich lässt sich der Mechanismus von Expansion durch Differenzierung bei der Lehrerbildung in einem doppelten Sinne nachweisen. Erstens ist das Lehrerbildungssystem selber diesem Prozess unterworfen. In der Konstitutionsphase der modernen Lehrerbildung in der ersten Hälfte des 19. Jahrhunderts wurden ausschliesslich seminaristische Ausbildungsgänge für Primarlehrerinnen und Primarlehrer geschaffen. Dieses Lehrerbildungssystem wurde in den letzten 150 Jahren in mehreren Schritten differenziert (Criblez, 2000a): Zunächst wurden Ausbildungsgänge für Sekundarlehrkräfte und für Gymnasiallehrkräfte an den Universitäten (z.B. in Zürich, Bern und Basel) geschaffen. Um die Jahrhundertwende folgten Ausbildungsgänge in den Frauenlehrberufen, also Ausbildungsgänge für Arbeitslehrerinnen, Kindergärtnerinnen und Hauswirtschaftslehrerinnen. Seit den 1950er-Jahren wurden Ausbildungsgänge für die Lehrkräfte der Volksschuloberstufe (Oberschulen, Realschulen, Primaroberstufen) geschaffen, zudem Ausbildungsgänge für Kleinklassenlehrkräfte. Insbesondere in der Expansionsphase seit Mitte der 1950er-Jahre, die mit einem grossen Lehrermangel verbunden war (Hodel, 1991), wurden neue Ausbildungsmodelle - zum Teil parallel zu den traditionell seminaristischen - geschaffen, Ausbildungsgänge im Anschluss an eine Matur und Ausbildungsgänge für Berufsleute. Insgesamt hat sich das Lehrerbildungssystem so differenziert, dass zu Beginn der 1990er-Jahre von einer «Vielfalt ohne Koordination» (Criblez, 1994) gesprochen werden musste. Von der momentanen Gründung Pädagogischer Hochschulen wird deshalb eine Integration der Lehrerbildung erwartet.

Diese Beispiele dokumentieren den Mechanismus Bildungsexpansion durch Systemdifferenzierung je einzeln an einem Teilbereich des Bildungssystem. Insgesamt kann von einem allgemeinen Mechanismus ausgegangen werden, der in Wachstumsphasen des Bildungssystem in der Regel eintritt ${ }^{18}$. Die Beispiele zeigen zudem, dass in der Regel auf eine Differenzierungsphase auch eine Integrationsphase folgt: «Das Bildungswachstum vollzieht sich nach dem Muster von Differenzierung und Integration» (Titze, 1999, S. 116). Dies gilt auch für die Sekundarstufe II, steht doch seit einiger Zeit die Zukunft der Sekundarstufe II als gesamter Bildungsstufe zur Diskussion, und die EDK hat kürzlich einen Bericht vorgelegt, der in Richtung Vereinheitlichung der Schultypen bzw. Integration der Sekundarstufe II zielt (EDK, 2000). 
Verfolgt man letztlich die Abfolge dieses Mechanismus von Differenzierung und Integration, so ist festzustellen, dass sich beide Prozesse im Bildungssystem von unten nach oben weiterentwickeln. Auf die Differenzierung der Sekundarstufe I folgt diejenige der Sekundarstufe II, anschliessend diejenige des tertiären Bildungsbereichs. Ebenso verläuft der Integrationsprozess, jedoch mit einem unterschiedlich langen Verzögerungseffekt gegenüber dem Differenzierungsprozess.

Wie sich diese Prozesse von Differenzierung und Integration im Bildungssystem auf die individuelle Wahrnehmung von Bildungschancen und damit auf die Bildungsbeteilgung ausgewirkt haben und weiter auswirken, ist heute für die Schweiz kaum erforscht. Dass die Bildungsexpansion die mit ihr verbundenen Erwartungen nach grösserer sozialer Gerechtigkeit kaum eingelöst hat, ist inzwischen bekannt (Lamprecht \& Stamm, 1996; Lévy et al., 1997). Becker kommt in einer neuen Studie zur Entwicklung in Deutschland sogar zum Schluss, dass sich die «Situation der ohnehin besonders benachteiligten Kinder eher verschlechtert als verbessert hat» (Becker, 2000, S. 466). Allein schon die Möglichkeit, dass dies auch für die Schweiz zutreffen könnte, würde rechtfertigen, über die sozialen Effekte von Differenzierung und Integration im Bildungssystem neu nachzudenken.

\section{Anmerkungen}

1 Der Beitrag konzentriert sich auf die Entwicklungen in der Schweiz. Bildungsexpansion ist jedoch ein internationales Phänomen. Auf die internationalen Bezüge wird v.a. im Abschnitt über die Hintergründe eingegangen. Selbstverständlich gibt es auch für die Reformen auf der Sekundarstufe II internationale Zusammenhänge und Vorbilder; auf sie kann an dieser Stelle nur sehr punktuell verwiesen werden.

2 Die Bildungsexpansionsphase der 1960er- und 70er-Jahre ist für die Schweiz bisher nur sehr punktuell wissenschaftlich aufgearbeitet worden; vgl. zur Expansion der Mittelschulen am Beispiel Zürichs den Beitrag von Andreas Rieger in diesem Heft, zur Dezentralisierung der Aargauer Mittelschulen Gretler (1993) und der Zürcher Mittelschulen Imhof, Delmore und Ottiger (1984).

3 Vgl. dazu die verschiedenen kantonalen Reformprojekte, die heute noch im Gange sind (im Raume der Nordwestschweiz zum Beispiel in den Kantonen Aargau, Basel-Landschaft oder Solothurn), aber auch den Bericht der Konferenz der kantonalen Erziehungsdirektoren zur Sekundarstufe I (EDK, 1995). Auch in Kantonen, in denen die Reform der Sekundarstufe I in Richtung Integration (im Sinne eines Abbaus von Systemdifferenzierung) abgeschlossen ist, wird die Diskussion um die Struktur dieser Schulstufe fortgesetzt (zum Beispiel im Kanton Basel-Stadt). Insgesamt ist diese Entwicklung der Sekundarstufe I wissenschaftlich kaum aufgearbeitet (Ausnahmen sind etwa Kussau, Oertel \& Wohlgemuth, 1998; Lüscher, 1998; Oertel, 1999).

4 Gleichstellung meint, dass die früheren Lateinauflagen für den Zugang zu den Medizinalprüfungen (und damit zum Medizinstudium) fallengelassen wurden.

5 Eine ähnliche Studie, wie sie hier für die Sekundarstufe II vorliegt, könnte für die Tertiärstufe erarbeitet werden. Neben dem Ausbau der Universitäten (quantitatives Wachstum, Differenzierung des Studienangebotes, Versuch von Neugründungen in den Kantonen Aargau, Luzern und Tessin) ist insbesondere auf die starke Ausbreitung der Höheren Technischen Lehranstalten, auf die Einführung von Abendtechnika, auf die Einführung der Höheren Wirtschafts- und Verwaltungsschulen sowie auf die Differenzierung der höhe- 
ren Berufsbildung durch die Revision des Berufsbildungsgesetzes von 1964 hinzuweisen. Als wichtigste Folge der Expansion im tertiären Bildungsbereich kann die allmähliche Entwicklung des Bildungssystem von einem hierarchisch gegliederten System mit einer Spitze zu einem hierarchisch gegliederten System mit zwei Spitzen (Universitäten und Fachhochschulen) gelten.

6 Die Zeitgeschichte hat sich in den letzten Jahren vermehrt mit den 1950er-, 60er- und 70er-Jahren zu beschäftigen begonnen. Für weitergehende Hintergründe sei auf folgende Darstellungen verwiesen: Blanc \& Luchsinger (1994), König et al. (1998), Leimgruber \& Fischer (1999) sowie Pfister (1995).

7 Ein Topos, der sich offensichtlich wiederholt. Rund fünfundzwanzig Jahre später publizierte die «National Commission on Excellence in Education» den Bericht "A Nation at Risk», welcher an der Schwelle zur Globalisierung der Wirtschaft das Schulsystem wiederum kritisierte, weil die Konkurrenzfähigkeit der amerikanischen Wirtschaft als gefährdet angesehen wurde (vgl. National Comission, 1984).

8 Vgl. Steiner (1971), die Sondernummer der Zeitschrift «Nachwuchsförderung» 1963 mit dem bezeichnenden Titel «Talenterfassung und Nachwuchsförderung» (Schweiz. Vereinigung, 1963) oder etwa - unter vielen andern - die beiden Aufsätze von Richard Meili "Was heisst Begabung und wo finden wir Begabte in der Schweiz?» (Meili, 1967) und Jacques Vontobel «Soziale Herkunft und Bildungschancen» (Vontobel, 1967).

9 Die Initiative wurde am 1. Oktober 1969 eingereicht und verlangte in Form einer allgemeinen Anregung vor allem eine verbesserte Schulkoordination in der Schweiz. Ähnliche Ziele verfolgten zwei Motionen in den eidgenössischen Räten. Die Schweiz. Konferenz der kantonalen Erziehungsdirektoren (EDK) verabschiedete Ende Oktober 1970 das Schulkonkordat, welches auf föderalistischer Grundlage Ähnliches bezweckte (Arnet, 2000, S. 17 ff.). Das Koordinationsanliegen kann u.a. als Folge des mit der Expansion verbundenen Differenzierungsprozesses interpretiert werden. Die Bestrebungen zur Schulkoordination der 1960er- und 70er-Jahre sind bislang historisch nicht aufgearbeitet.

10 Der Vollständigkeit halber sei erwähnt, dass die Bundesverfassung bereits 1963 durch einen Stipendienartikel ergänzt worden war (Art. 27quater), der es dem Bund erlaubte, sich in der Nachwuchsförderung auch auf diesem Wege zu engagieren.

11 Eine Analyse der damaligen Hochschul- und Forschungspolitik fehlt bislang; einen kurzen Überblick gibt Horvath (1998).

12 Einen Überblick über die Entwicklungen geben Egger (1978) und Erziehungsrat (1964).

131956 hatte bereits Karl Käfer, Professor für Handelswissenschaften an der Universität Zürich, mit einer von der Schweizerischen Handelsschul-Rektorenkonferenz und der Schweizerischen Gesellschaft für Kaufmännisches Bildungswesen eingesetzten Kommission einen ausführlichen Plan für eine allgemeinbildende Mittelschule mit Schwerpunkt Sozialwissenschaften vorgelegt (Kommission, 1957).

14 Der Verein Schweizerischer Gymnasiallehrer (VSG) hatte sich erstmals an seiner Studienwoche 1967 mit dem Thema beschäftigt (vgl. VSG, 1968, S. 76 ff.).

15 Einen - zwar nicht mehr ganz aktuellen - Überblick über die Berufsbildung in der Schweiz geben Wettstein et al. (1985); zur Geschichte der Berufsbildung vgl. die beiden Überblicksdarstellungen von Wettstein (1987) und Tabin (1989).

16 Einen Überblick über die Entwicklung der Berufsmittelschule geben Feierabend, 1975; Hässler, 1977; Sommerhalder, 1989.

17 Gewerkschaften und linke Parteien lehnten jedoch die definitive Einführung der Berufsmittelschule im Berufsbildungsgesetz ab. Sie waren der Meinung, dass der Berufsschulunterricht nicht nur für eine kleine Elite wesentlich verbessert werden sollte. Von Seiten der Arbeitgeberverbände war die durch den Bund institutionalisierte Regelung der Anlehre abgelehnt worden; sie sollte den Bedürfnissen der Betriebe und der Jugendlichen angepasst werden können (Botschaft Berufsbildungsgesetz, 1977, S. 710, 716 ff.). 
18 Lundgreen unterscheidet vier Typen der Differenzierung: die funktionale Differenzierung zwischen Institutionen, die funktionale Differenzierung innerhalb von Institutionen, die hierarchische Differenzierung innerhalb von Institutionen und die hierarchische Differenzierung zwischen Institutionen (Lundgreen, 2000, S. 141).

\section{Literatur}

Ad hoc-Arbeitsgruppe Diplommittelschulen (1983). Diplommittelschulen (DMS). Stand der Entwicklung. Genf: EDK.

Arbeitsgruppe Perspektivstudien (1971). Perspektiven des schweiz. Bildungswesens. St. Gallen: s.n.

Arbeitsausschuss zur Förderung des wissenschaftlichen und technischen Nachwuchses (1959). Schlussbericht. Bern: s.n.

Arnet, M. (2000). Das Schulkonkordat vom 29. Oktober 1970. Entstehung - Geschichte - Kommentar. Bern: EDK.

Barth, A. (1919). Die Reform der höheren Schulen in der Schweiz. Untersuchungen und Vorschläge über die Maturitätsverhältnisse und andere Mittelschulfragen. Basel: Kober.

Becker, R. (2000). Bildungsexpansion und Bildungsbeteiligung - Oder: Warum immer mehr Schulpflichtige das Gymnasium besuchen. Zeitschrift für Erziehungswissenschaft, 3, 447480.

Blanc, J.-D. \& Luchsinger, Ch. (Hrsg.) (1994). achtung: die 50er Jahre! Annäherungen an eine widerspüchliche Zeit. Zürich: Chronos.

Bonner, Th. N. (1958). Sputniks and the Educational Crisis in America. Journal of Higher Education, 29, 177-184, 232.

Botschaft Berufsbildungsgesetz (1977). Botschaft zu einem neuen Bundesgesetz über die Berufsbildung vom 26. Januar 1977. Schweizerisches Bundesblatt, 129, (1), 681-766.

Botschaft Fachhochschulgesetz (1994). Botschaft zu einem Bundesgesetz über die Fachhochschulen (Fachhochschulgesetz, FHSG) vom 30. Mai 1994. Bern: EDMZ.

Botschaft Bildung (1998). Botschaft über die Förderung von Bildung, Forschung und Technologie in den Jahren 2000-2003 vom 25. November 1998. Bern: EDMZ.

Chresta, H. (1970). Aktuelle Probleme der Berufsbildung. Zürich: Kantonales Amt für Berufsbildung.

Criblez, L. (1994). Lehrerbildung in der Schweiz: Vielfalt ohne Koordination? Bildungsforschung und Bildungspraxis, 16, 139-160.

Criblez, L. (2000a). Das Lehrerseminar - Zur Entwicklung eine Lehrerbildungskonzeptes. In L. Criblez, R. Hofstetter \& D. Périsset Bagnoud (Hrsg.), La formation des enseignant(e)s primaires - Die Ausbildung von PrimarlehrerInnen (S. 298-338). Bern: Lang.

Criblez, L. (2000b). Das Gymnasium im Stress. Magazin für Schule und Kindergarten, (118), $15-24$.

Dahrendorf, R. (1965). Bildung ist Bürgerrecht. Plädoyer für eine aktive Bildungspolitik. o.O.: Nannen.

Dellsperger, H. (1968). Stehen wir in unserer Berufsbildung vor bedeutenden Wandlungen? Schweizerische Blätter für Gewerbeunterricht, 93, 193-197.

Deppeler, R. (1967). High School und Gymnasium. Zwei Welten. Schweizerische Zeitschrift für Nachwuchs und Ausbildung, 6, 176-191.

Douglas, J. A. (2000). A Certain Future: Sputnik, Higher Education, and the Survival of a Nation. In R. D. Launius, J. M. Logsdon \& R. W. Smith (Eds.), Reconsidering Sputnik. Forty Years Since the Soviet Satellite (S. 327-363). Amsterdam: Harwood academic publishers. 
Dubs, R. (1968). Das Wirtschaftsgymnasium. Ein Beitrag zu den Problemen eines neuen Mittelschultypus aus schweizerischer Sicht. Zürich \& St. Gallen: Polygraphischer Verlag.

Durtschi, G. (1963). Die Problematik des Zweiten Bildungsweges. Nachwuchsförderung, 2, (4), 12-16.

EDK (1976/1995). Empfehlungen und Beschlüsse zum Bericht «Mittelschule von morgen» vom 11. März 1976. In EDK, Empfehlungen und Beschlüsse (S. 37-38). Bern: EDK.

EDK (1995). Perspektiven für die Sekundarstufe I. Bern: EDK.

EDK (2000). Die Sekundarstufe II hat Zukunft. Bern: EDK.

Egger, E. (1978). Die Reformetappen 1946 bis 1978. In A. Vonlanthen, U. P. Lattmann \& E. Egger, (1978), Maturität und Gymnasium. Ein Abriss über die Entwicklung der eidgenössischen Maturitätsordnungen und deren Auswirkungen auf das Gymnasium (S. 101-130). Bern: Haupt.

Eidg. Expertenkommission für Fragen der Hochschulförderung (1964). Bericht. Bern: EDMZ.

Eidg. Kommission für Nachwuchsfragen auf dem Gebiete der Geisteswissenschaften und der medizinischen Berufe sowie des Lehrerberufes auf der Mittelschulstufe (1963). Bericht. Bern: EDMZ.

Erziehungsrat des Kantons Zürich (1964). Aktuelle Mittelschulfragen unter besonderer Berücksichtigung der Stellung des Maturitätstypus C. Zürich: [Erziehungsdirektion].

Expertenkommission zum Studium der Mittelschule von morgen (1972). Mittelschule von morgen. Bericht. Frauenfeld: Huber.

Faerber, H. R. (1966). Bildungsinflation am Gymnasium? Schweizerische Zeitschrift für Nachwuchs und Ausbildung, 5, (1), 27-31.

Feierabend, U. J. (1975). Die Berufsmittelschule mit besonderer Berücksichtigung der Verhältnisse im Kanton Solothurn. Aarau: Sauerländer.

Gretler, A. (1993). Die Dezentralisierung der Mittelschulen im Aargau - Von der Aargauischen Kantonsschule Aarau zur Aargauischen Maturitätsschule für Erwachsene. In B. Biberstein, S. Brändli \& F. Vogt (Hrsg.), Aufklärung bis in die Niederungen. Politik im Schweizer Mittelland (S. 89-109). o.O.: s.n.

Grossenbacher, F. (1968). Eine Schule für mittlere Kader? Schweizerische Zeitschrift für Gewerbeunterricht, 93, 103-107.

Häsler, A. A. (Hrsg.). (1967). Schulnot im Wohlstandsstaat. Zürch: Ex Libris.

Hässler, T. (1977). Die Berufsmittelschule im industriell-gewerblichen Bereich. Berechtigung Zielsetzung - Konzept. Winterthur: Schellenberg.

Hess, F. (1966). Überschätzung der höheren Ausbildung? Schweizerische Zeitschrift für Nachwuchs und Ausbildung, 5, (1), 37-40.

Hess, F, Latscha, F. \& Schneider, W. (1966). Die Ungleichheit der Bildungschancen. Soziale Schranken zur Höheren Bildung. Olten \& Freiburg/Br.: Walter.

Hodel, G. (1991). LehrerInnenmangel - LehrerInnenarbeitslosigkeit. Bern: Institut für Pädagogik (unveröffentlichte Lizentiatsarbeit).

Horvath, F. (1998). Im Windschatten der Wissenschaftspolitik. Ständisch-föderalistische Interessenentfaltung im Zeichen des «Bildungsnotstandes». In M. König, G. Kreis, F. Meister \& G. Romano (Hrsg.), Dynamisierung und Umbau. Die Schweiz in den 60er und 70er Jahren (S. 81-93). Zürich: Chronos.

Imhof, B., Delmore, S. \& Ottiger, M. (1984). Die Zürcher Mittelschulen 1833-1983. 150 Jahre Schulentwicklung im Spiegel der Schülerzahlen, Schulen und Schultypen. Zürich: Erziehungsdirektion.

Jenzer, C. (1998). Schulstrukturen als historisch-gewachsenes Produkt bildungspolitischer Vorstellungen. Bern: Lang.

Jermann, M. (1972). Bildungswesen, Arbeitsmarkt und Wirtschaftswachstum. Bern \& Stuttgart: Haupt. 
Kommission zur Revision des Normallehrplanes [1957]. Vorschlag zur Schaffung von Gymnasien wirtschafts- und sozialwissenschaftlicher Richtung als Ziel der Reform der Maturitätsabteilungen unserer Handelsmittelschulen samt Lehrplan und Stundentafel. [Zürich: Schweizerische Gesellschaft für Kaufmännisches Bildungswesen].

König, M., Kreis, G., Meister, F. \& Romano, G. (Hrsg.) (1998). Dynamisierung und Umbau. Die Schweiz in den 60er und 70er Jahren. Zürich: Chronos.

KSGR [Konferenz der schweizerischen Gymnasialrektoren] (1948). Gegenwartsfragen des Gymnasiums. Aarau: Sauerländer.

Kussau, J., Oertel, L. \& Wohlgemuth, A. (1998). Reform und Immobilismus im Schulwesen. Eine Politikstudie über Strukturreformen anhand der Kantone Basel-Stadt, Bern, Genf, Tessin und Zürich. Zürich: s.n. [Polykopie].

Lamprecht, M. \& Stamm, H. (1996). Soziale Ungleichheit im Bildungswesen. Bern: Bundesamt für Statistik.

Launius, R. D., Logsdon, J. M. \& Smith, R. W. (Eds.) (2000). Reconsidering Sputnik. Forty Years Since the Soviet Satellite. Amsterdam: Harwood academic publishers.

Leimgruber, W. \& Fischer, W. (Hrsg.) (1999). «Goldene Jahre». Zur Geschichte der Schweiz seit 1945. Zürich: Chronos.

Lévy, R., Joye, D., Guye, O. \& Kaufmann, V. (1997). Tous égaux? De la stratification aux représentations. Zürich: Seismo.

Lundgreen, P. (2000). Schule im 20. Jahrhundert. Institutionelle Differenzierung und expansive Bildungsbeteiligung. Zeitschrift für Pädagogik, 42. Beiheft, 140-165.

Lüscher, L. (1997). Geschichte der Schulreform in der Stadt Bern von 1968 bis 1988. Bern: Lang.

Meili, R. (1967). Was heisst Begabung, und wo finden wir Begabte in der Schweiz? Schweizerische Lehrerzeitung, 112, 759-763

Meylan, J.-P. (1996). Die Erneuerung des Gymnasiums und die Anerkennung der Maturitäten. Stationen der Debatte 1968-1995. In EDK (Hrsg.), Von der «Mittelschule von morgen» zur Maturitätsreform 1995 (S. 7-45). Bern: EDK.

Meylan, J.-P. \& Ritter, V. (1985). Das schweizerische Gymnasium. Wachstum und Reformen der letzten 15 Jahre. In EDK (Hrsg.), Materialien zur Entwicklung des Mittelschulunterrichts (S. 28-43). Bern: EDK.

National Commission on Excellence in Education (1984). A Nation at Risk. Portland: USA Research.

Neue Helvetische Gesellschaft (1961). Probleme des wissenschaftlichen und technischen Nachwuchses. Zürich: Atlantis.

Nyikos, L. (1967). Eine Schule für mittlere Kader? gymnasium helveticum, 22, (1), 32-46.

Nyikos, L. (1968a). Allgemeine Berufsmittelschule. Schweizerische Blätter für Gewerbeunterricht, 93, 99-103.

Nyikos, L. (1968b). Bildung ist kein Vorrecht der Gymnasiasten. Schweizerische Lehrerzeitung, 113, 1063-1067.

OECD (1967). Begabung und Bildungschancen. Frankfurt/M.: Diesterweg.

Oertel, L. (1997). Schulreform - ein Zürcher Politikversuch. Zürich: Orell Füssli.

Panchaud, G. (1966). Le niveau des élèves est-il en baisse? Schweizerische Zeitschrift für Nachwuchs und Ausbildung, 5, (1), 32-36.

Pfister, Ch. (Hrsg.) (1995). Das 1950er Syndrom. Der Weg in die Konsumgesellschaft. Bern: Haupt.

Picht, G. (1964). Die deutsche Bildungskatastrophe. Olten: Walter.

Räber, L. (1969). Bildung und Gymnasium. Weinheim, Berlin \& Basel: Beltz.

Schaub, P. (1966). Der zweite Bildungsweg - eine zeitgemässe Bildungsmöglichkeit? Schweizerische Zeitschrift für Nachwuchs und Ausbildung, 5, 225-230.

Schweizerischer Wissenschaftsrat (1967). Ausbau der Schweizerischen Hochschulen. Bern: [Schweiz. Wissenschaftsrat]. 
Schweizerischer Wissenschaftsrat (1972). Zweiter Bericht über den Ausbau der schweizerischen Hochschulen. Bern: Schweizerischer Wissenschaftsrat.

Schweizerischer Wissenschaftsrat (1978). Dritter Bericht über den Ausbau der schweizerischen Hochschulen. Beitrag zu einer bildungspolitischen Gesamtperspektive. Bern: Schweizerischer Wissenschaftsrat.

Schweiz. Vereinigung für die Förderung des beruflichen und wissenschaftlichen Nachwuchses (Hrsg.) (1963). Talenterfassung und Nachwuchsförderung. Zürich: s.n.

Sommerhalder, P. (1968). Berufsbildung im Umbruch. Schweizerische Blätter für Gewerbeunterricht, 93, 225-230.

Sommerhalder, P. (1970). Berufsbildung im Umbruch. Zürich: Kantonales Amt für Berufsbildung.

Sommerhalder, P. (1989). So entstand die Berufsmittelschule (BMS). Zürich: Institut für Bildungsforschung und Berufspädagogik im Amt für Berufsbildung des Kantons Zürich.

Steiner, H. (1971). Nachwuchsförderung für akademische Berufe. Pädagogische, kulturpolitische und begabungstheoretische Aspekte der Begabtenförderung. Bern: Haupt.

Stettler, N. (1994). «Die Zukunft ist errechenbar ...». In D. Blanc \& Ch. Luchsinger (Hrsg.), achtung: die 50er Jahre! (S. 95-117). Zürich: Chronos.

Studienkommission der Konferenz Schweizerischer Gymnasialrektoren (1965). Gymnasialrektoren besuchen amerikanische Schulen. Bericht über eine Studienreise durch die USA. Aarau: Sauerländer.

Studienkommission Diplommittelschulen (1977). Diplommittelschulen. Genf: EDK.

Studienkommission für Typenfragen (1961). Wege gymnasialer Bildung. Arbeitsbericht der Studienkommission für Typenfragen. Aarau: Sauerländer.

Studienkommission zur Überprüfung des Unterrichtsauftrages der gewerblich-industriellen Berufsschule (1968). Ergebnisse der Beratungen. Schweizerische Zeitschrift für Gewerbeunterricht, 93, 295-297.

Tabin, J.-P. (1989). Formation professionnelle en Suisse. Histoire et actualité. Lausanne: réalités sociales.

Titze, H. (1999). Wie wächst das Bildungssystem? Zeitschrift für Pädagogik, 45, 103-120.

Van Dyke, G. J. (2000). Sputnik: A Political Symbol and Tool in 1960 Campaign Politics. In R. D. Launius, J. M. Logsdon \& R. W. Smith (Eds.), Reconsidering Sputnik. Forty Years Since the Soviet Satellite (S. 365-400). Amsterdam: Harwood academic publishers.

Vontobel, J. (1967). Soziale Herkunft und Bildungschancen. Schweizerische Lehrerzeitung, 112, 895-901.

VSG [Verein Schweizerischer Gymnasiallehrer] (1968). Die Mittelschule von morgen. Aarau: Sauerländer.

Wegleitung (1970/1977). Wegleitung für die Errichtung und die Organisation von Berufsmittelschulen und deren Subventionierung durch den Bund vom 26. Juni 1970. In T. Hässler, Die Berufsmittelschule im industriell-gewerblichen Bereich (S. 160-167).Winterthur: Schellenberg.

Widmaier, P. (1966). Bildungsplanung. Ansätze zu einer rationalen Bildungspolitik. Stuttgart: Klett.

Widmer, K. (1976). Bildungsplanung und Schulreform. Frauenfeld \& Stuttgart: Haupt.

Zweifel, O. (1967). Massnahmen zur Mittelschulförderung. Gefahren der Qualitätseinbusse bei ansteigender Maturandenquote. gymnasium helveticum, 22, (2), 106-124.

Wettstein, E. (1987). Die Entstehung der Berufsbildung in der Schweiz. Aarau: Sauerländer.

Wettstein, E., Bossy, R., Dommann, F. \& Villiger, D. (1985). Die Berufsbildung in der Schweiz. Eine Einführung. Schaffhausen: Deutschschweizerische BerufsbildungsämterKonferenz. 


\section{L'expansion des systèmes de formation à travers leur différenciation, I'exemple du secondaire II}

Résumé

Dans cette contribution, la thématique de l'expansion des systèmes de formation sera analysée non sous ses aspects quantitatifs (accroissement du nombre d'élèves, d'écoles, d'enseignants, allongement des parcours de formation), mais d'un point de vue institutionnel. Cette analyse sera centrée sur la différenciation au degré secondaire II. D'une part, l'arrière plan de cette différenciation sera mis en lumière, d'autre part, le processus même de différenciation sera reconstitué, aussi bien au niveau des gymnases qu'à celui de la formation professionnelle. Dans ces deux domaines, l'expansion est liée à des formes différentes de différenciation.

Pour terminer, cet article montrera que l'expansion par différenciation appartient à un phénomène de croissance plus général que l'on observe dans le système éducatif et qui ne se limite ni à la période 1960 - 1970, ni au degré secondaire II.

\section{L'espansione della formazione attraverso la differenziazione del sistema scolastico - l'esempio del secondario II}

\section{Riassunto}

In questo contributo l'espansione della formazione non viene analizzata tanto sotto il profilo quantitativo (più allievi, più scuole, più insegnanti e percorsi formativi più lunghi) quanto dal punto di vista della strutturazione istituzionale. La differenziazione del secondario II è collocata al centro dell'interesse. Da un lato si ricostruisce il processo di differenziazione del sistema sia dei ginnasi che delle scuole professionali, dall'altro lato se ne mettono a fuoco le ragioni. In entrambi i settori l'espansione è connotata da differenti forme di differenziazione. Infine si mostra che il fenomeno dell'espansione attraverso la differenziazione vale in generale per le fasi di sviluppo dei sistemi scolastici e quindi non è circoscrivibile né gli anni '60 e '70 né al secondario II.

\section{Educational expansion through a differentiation of the educational system: an example of the middle secondary school level (eighth grade.)}

\section{Summary}

In this contribution, the expansion of education is analyzed in terms of its institutional characteristics rather than its quantitative characteristics (more students, more schools, more teachers, longer educational careers. The differentiation of the middle secondary level is its primary interest. On the one hand, the 
background is illuminated; on the other hand, the process of differentiation of the system for Gymnasiums as well as trade schools is reconstructed. In both sections of the educational system, the expansion is interconnected with various differentiations of the system. To conclude, it becomes apparent that we are dealing with the phenomenon of expansion through the differentiation of the system that is a general sign of growth in the educational system, which does not merely concern the 60's and 70's or just the middle secondary school level. 